\title{
Method for Molecular Layer Deposition Using Gas Cluster Ion Beam Sputtering with Example Application In Situ Matrix-Enhanced Secondary Ion Mass Spectrometry
}

\section{- Supporting Information -}

\author{
Matthias Lorenz, ${ }^{* \dagger}$ Junting Zhang, ${ }^{\dagger}$ Alexander G. Shard,$^{\dagger}$ Jean-Luc Vorng ${ }^{\dagger}$, Paulina D.
} Rakowska, ${ }^{\dagger}$ Ian S. Gilmore ${ }^{* \dagger}$

${ }^{\dagger}$ National Physical Laboratory, Hampton Road, Teddington TW11 0LW, United Kingdom

\section{Table of Contents}

Figure SI-1. Chemical structures of the compounds used in the study ................................... 2

Figure SI-2. Angular Distribution of Ejection Probabilities .................................................... 3

Figure SI-3. ME SIMS Multimodal Utilization of 2,5-DHB and $\alpha-\mathrm{CHCA}$............................. 4

Figure SI-4. 2,5-DHB ME SIMS - Low $m / z$ Range complementing data in Figure 3 ................. 5

Figure SI-5. Effect of increasing DHB thickness (inferred from the sputtering ion fluence) on the SIMS intensity for the DHB matrix $(m / z, 137)$ and AM $(m / z, 646)$........................................ 6

Table S1. Overview of experiments on the sputter-deposition of molecular matrix compounds... 7

Table S2. Overview of experiments on the signal enhancement using molecular matrix compounds

Table S3. Enhancement ratio of amiodarone peak at $m / z 646.00$ from 15 cycles of in situ ME SIMS

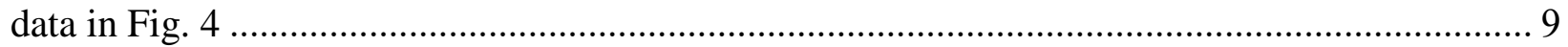

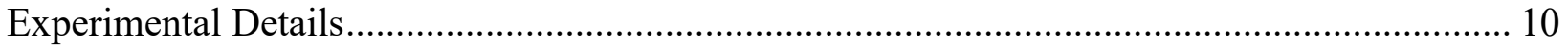




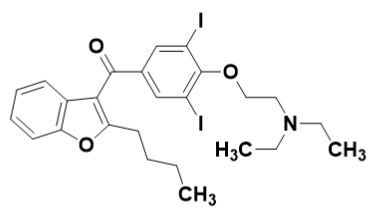

Amiodarone

Chemical Formula: $\mathrm{C}_{25} \mathrm{H}_{29} \mathrm{I}_{2} \mathrm{NO}_{3}$ Exact Mass: 645.02368

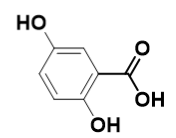

4,5-Dihydroxybenzoic acid Chemical Formula: $\mathrm{C}_{7} \mathrm{H}_{6} \mathrm{O}_{4}$ Exact Mass: 154.02661

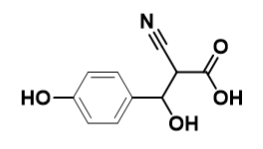

$\alpha$-cyano-4-hydroxycinnamic acid Chemical Formula: $\mathrm{C}_{10} \mathrm{H}_{9} \mathrm{NO}_{4}$ Exact Mass: 207.05316

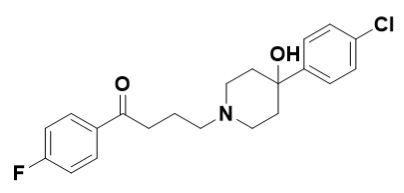

Haloperidol

Chemical Formula: $\mathrm{C}_{21} \mathrm{H}_{23} \mathrm{ClFNO}_{2}$ Exact Mass: 375.14013

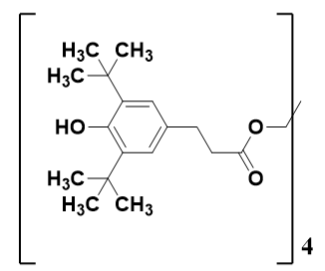

Irganox 1010

Chemical Formula: $\mathrm{C}_{73} \mathrm{H}_{108} \mathrm{O}_{12}$ Exact Mass: 1176.78408
$\mathrm{H}_{3} \mathrm{C}$

$\mathrm{H}_{3} \mathrm{C} \sim \mathrm{N}$

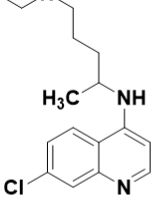

Chloroquine

Chemical Formula: $\mathrm{C}_{18} \mathrm{H}_{26} \mathrm{ClN}_{3}$ Exact Mass: 319.18153

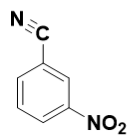

3-Nitrobenzonitrile

Chemical Formula: $\mathrm{C}_{7} \mathrm{H}_{4} \mathrm{~N}_{2} \mathrm{O}_{2}$

Exact Mass: 148.02728

Figure SI-1. Chemical structures of the compounds used in the study. 

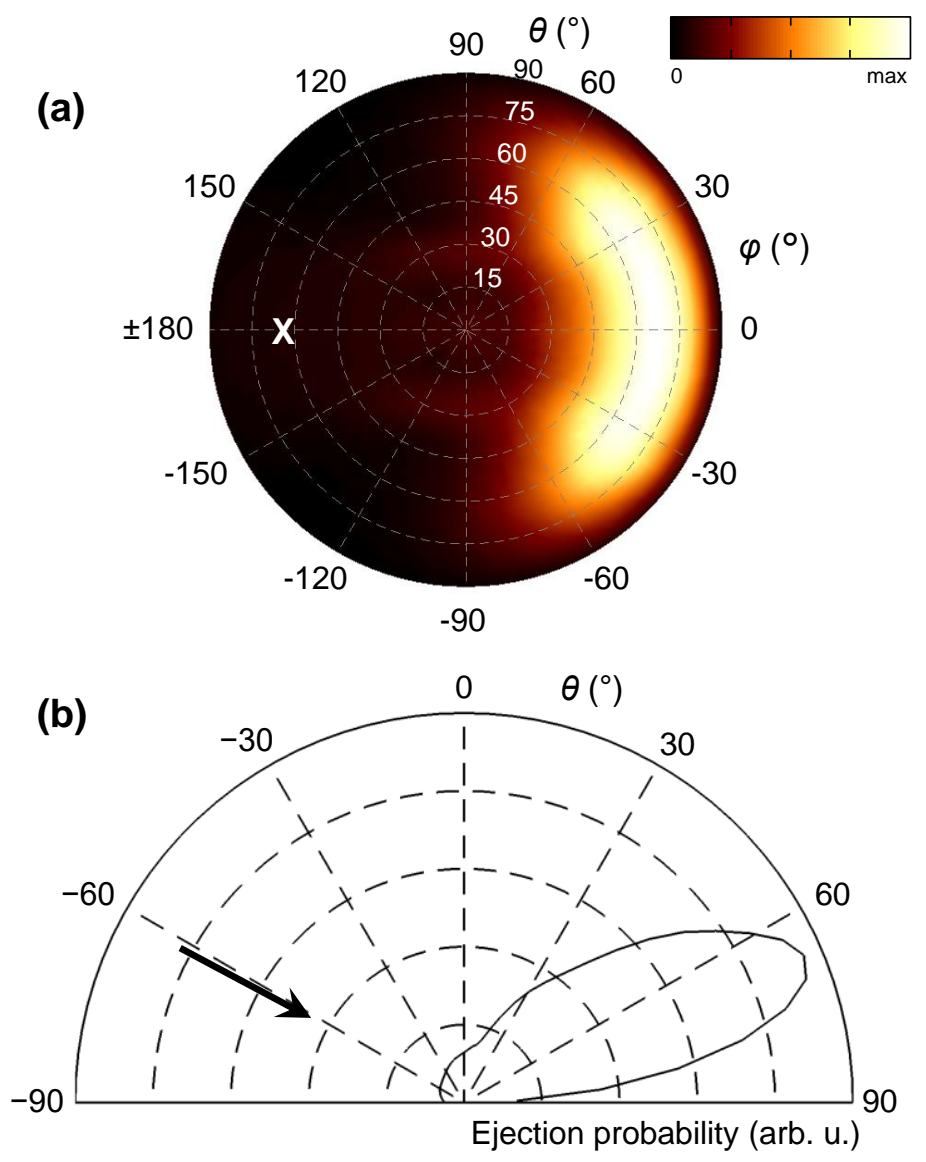

Figure SI-2. Angular Distribution of Ejection Probabilities: (a) Spherical diagram with full polar $(\theta)$ and azimuthal $(\varphi)$ angular description of the ejection probability for sputtered molecules as derived from extrapolation of experimental data published by Lorenz et al., Ref. 32. Data describe the sputtering of Irganox 1010 with $10 \mathrm{keV} \mathrm{Ar}_{2000}$ GCIB projectiles at $62^{\circ}$ angle of incidence (relative to normal); (b) Polar distribution of ejection probabilities vs. polar angle $(\theta)$ for the plane that contains the primary ion beam (azimuthal angle $\varphi=0^{\circ}$ ). The plots are normalized to individual scales. 

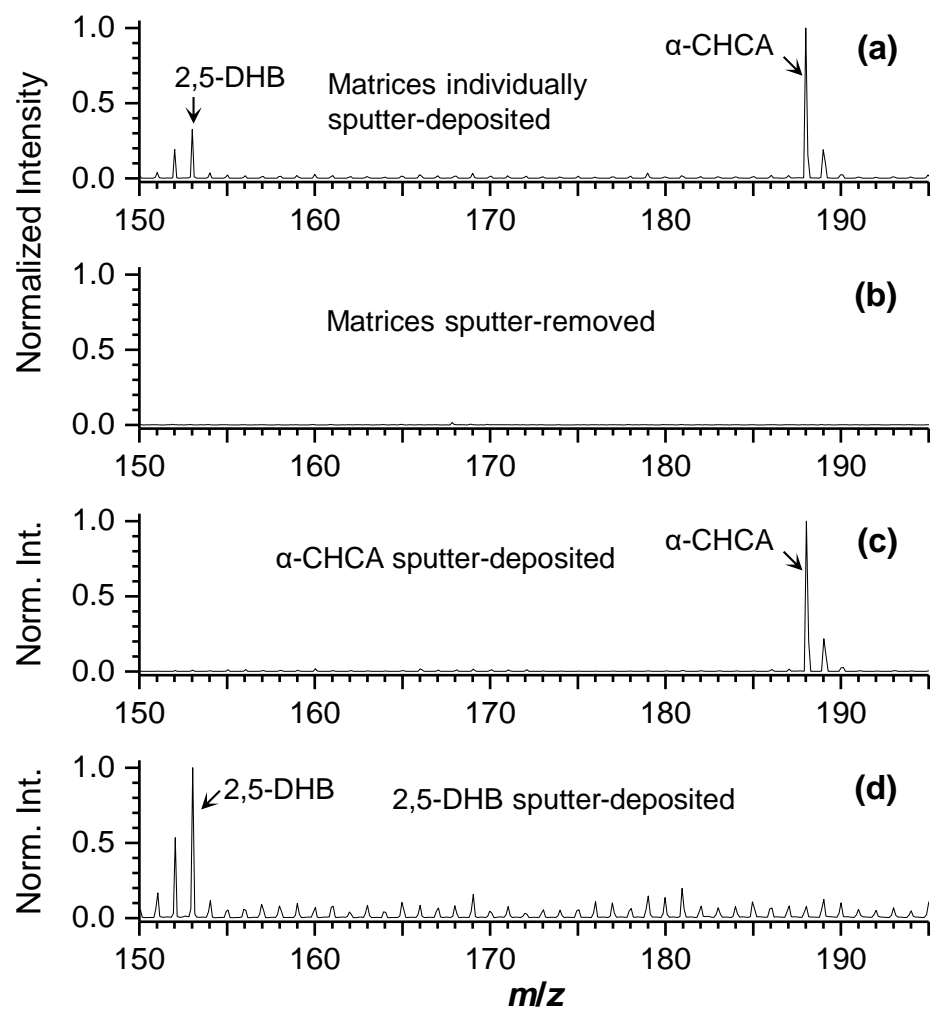

Figure SI-3. ME SIMS Multimodal Utilization of 2,5-DHB and $\alpha$-CHCA: (a) Negative polarity SIMS spectrum showing signals for sputter-deposited 2,5-DHB and $\alpha$-CHCA, collected on a cleaned silicon wafer. Both species were individually and independently sputter deposited from separate matrix reservoirs; (b) Negative polarity SIMS spectrum of the same surface after sputtercleaning by GCIB erosion. The spectra in (a) and (b) are normalized to a common scale; (c, d) Negative polarity SIMS spectra of the individually sputter-deposited compounds, $\alpha$-CHCA and 2,5-DHB, respectively. A sputter-cleaning cycle was used to clean the silicon sample surface between both experiments. The spectra in (c) and (d) are normalized to individual scales. 

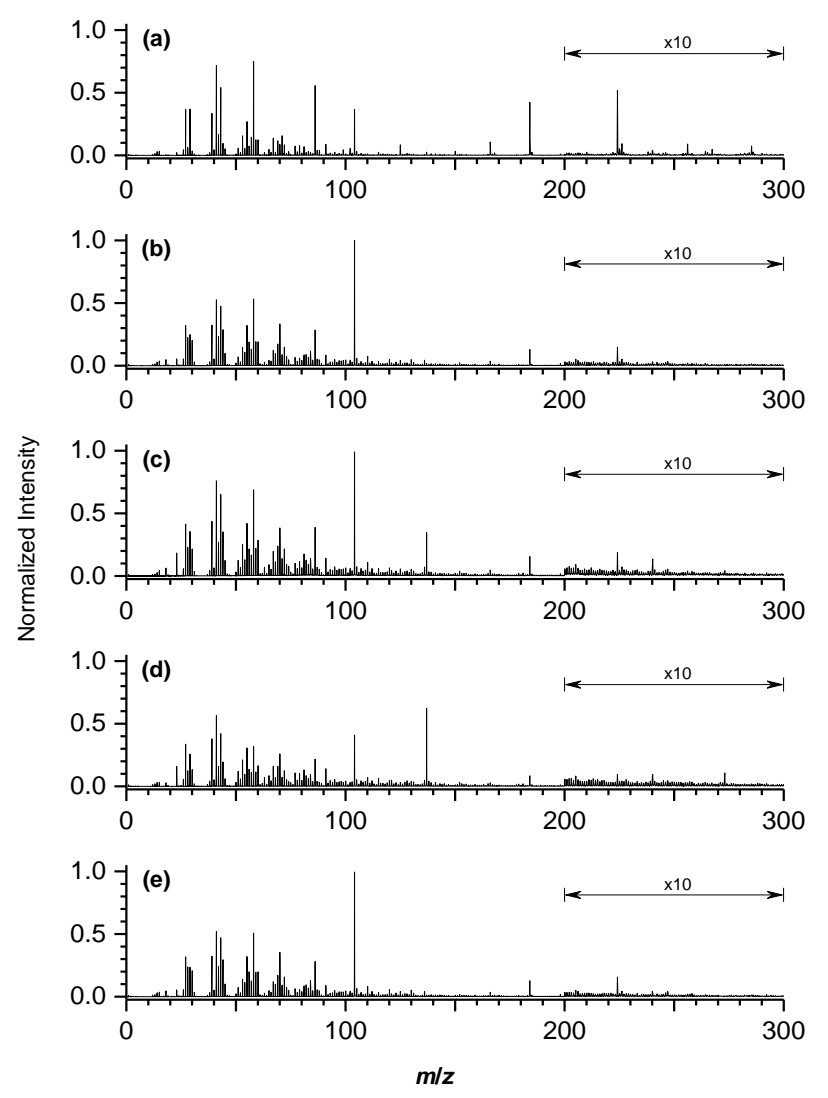

Figure SI-4. 2,5-DHB ME SIMS - Low $\mathrm{m} / \mathrm{z}$ Range complementing data in Figure 3 of the manuscript: (a) Positive polarity SIMS spectrum for an aged sample of chloroquine (CQ) at $\mathrm{m} / z$ $319 \mathrm{Da}$, haloperidol (HP) at $m / z 375 \mathrm{Da}$, and amiodarone (AM) at $m / z 645 \mathrm{Da}$ in tissue homogenate; (b) Positive polarity SIMS spectrum for the sample after sputter-cleaning of the aged top layer of the sample; (c) Spectrum of the in operando sputter deposition of 2,5-DHB; (d) Spectrum acquired following the in situ sputter deposition of 2,5-DHB over a period of $15 \mathrm{~min}$; (e) spectrum acquired after sputter-cleanup of the analytical surface area. All experiments were performed at room temperature, from the same sample area. 
(a)

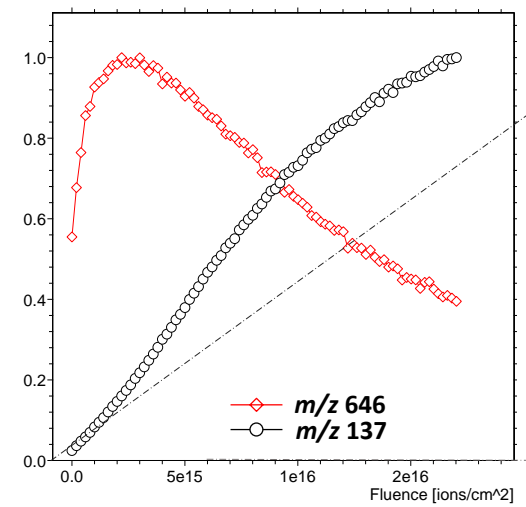

(b)

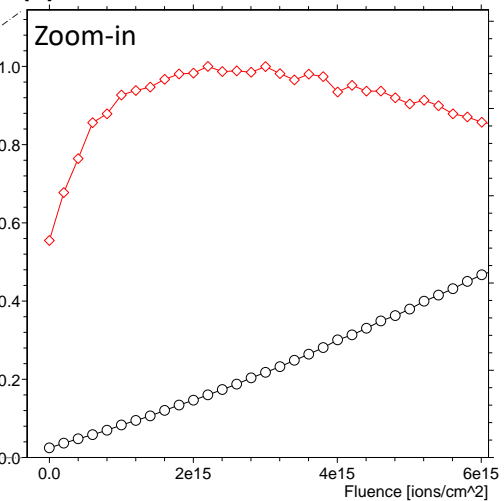

Figure SI-5. Effect of increasing 2,5-DHB thickness (inferred from the sputtering ion fluence) on the SIMS intensity for the 2,5-DHB matrix $(\mathrm{m} / \mathrm{z}, 137)$ and AM $(\mathrm{m} / \mathrm{z} 646)$. The positive polarity SIMS data are normalized to the respective maximum intensities for the $\mathrm{m} / \mathrm{z}, 137$ and $\mathrm{m} / \mathrm{z} 647$ ions. 
Table S1. Overview of experiments on the sputter-deposition of molecular matrix compounds.

\begin{tabular}{|l|l|l|}
\hline Parameter & Figure 2c & Figure 2d \\
\hline Matrix (model) & Irganox 1010 & 2,5-DHB \\
\hline Sample temp. & Room temperature (RT) \\
\hline Matrix temp. & RT \\
\hline Sample material & Si wafer & \multicolumn{2}{|l}{$\operatorname{Ar}_{2500}(7.0 \mathrm{nA})$} \\
\hline Sample structure & Topmount & \\
\hline GCIB (10 keV) & Ar $5000(1.6 \mathrm{nA})$ & \\
\hline Sample clean-up & None \\
\hline Matrix sputtering & 140 min \\
\hline Matrix clean-up & $\mathrm{n} / \mathrm{a}$ \\
\hline LMIG & $\mathrm{n} / \mathrm{a}$ \\
\hline Ion polarity & $\mathrm{n} / \mathrm{a}$ \\
\hline
\end{tabular}


Table S2 Overview of experiments on the signal enhancement using molecular matrix compounds.

\begin{tabular}{|c|c|c|c|c|}
\hline Parameter $^{\mathbf{a}}$ & Figure 3 & Figure 4 & Figure 5 & Figure 6 \\
\hline Ma. (model) & $2,5-\mathrm{DHB}$ & $2,5-\mathrm{DHB}$ & $10 \%$ FA (s) & 3-NBN \\
\hline Sa. temp. & RT & RT & $\leq 10^{\circ} \mathrm{C}^{\mathrm{b}}$ & $\leq 10^{\circ} \mathrm{C}^{\mathrm{b}}$ \\
\hline Ma. temp. & RT & RT & $-120 \pm 2{ }^{\circ} \mathrm{C}$ & $-108 \pm 2{ }^{\circ} \mathrm{C}$ \\
\hline Sa. material & $\begin{array}{l}\mathrm{CQ} / \mathrm{AM} / \mathrm{HP} \text { in bovine } \\
\text { liver tissue standard }\end{array}$ & $\begin{array}{l}\text { AM in bovine liver } \\
\text { tissue standard }\end{array}$ & \multicolumn{2}{|c|}{$\mathrm{CQ} / \mathrm{AM} / \mathrm{HP}$ in bovine liver tissue standard } \\
\hline Sa. structure & Topmount & Topmount & \multicolumn{2}{|c|}{ Cold stage } \\
\hline GCIB (10 keV) & $\operatorname{Ar}_{2500}(7.0 \mathrm{nA})$ & $\operatorname{Ar}_{2500}(8.0 \mathrm{nA})$ & \multicolumn{2}{|c|}{$\operatorname{Ar}_{5000}(1.6 \mathrm{nA})$} \\
\hline Sa. clean-up & None & 15 scans & \multicolumn{2}{|l|}{$60 \mathrm{~s}$} \\
\hline Ma. sputt. & $15 \mathrm{~min}$ & 30 scans per cycle & $10 \mathrm{~min}$ & $5 \mathrm{~min}$ \\
\hline Ma. clean-up & $60 \mathrm{~s}$ & 15 scans per cycle $^{\mathrm{c}}$ & \multicolumn{2}{|l|}{ none } \\
\hline LMIG & \multicolumn{2}{|c|}{$30 \mathrm{keV} \mathrm{Bi}_{3}^{+}(0.15 \mathrm{pA})$} & \multicolumn{2}{|c|}{$25 \mathrm{keV} \mathrm{Bi}_{3}^{+}(0.12 \mathrm{pA})$} \\
\hline Ion polarity & \multicolumn{4}{|c|}{ Positive } \\
\hline
\end{tabular}

${ }^{a}$ see Table 1 for abbreviations; ${ }^{b}$ reduced temperature due to imperfect thermal insulation. ${ }^{c}$ For figure 4, 15 scans include matrix clean-up and sample erosion. 
Table S3 Enhancement ratio of the amiodarone peak at $\mathrm{m} / \mathrm{z} 646.00$ from 15 cycles of in situ ME SIMS data in Fig. 4, demonstrating the cycle repeatability of the method.

\begin{tabular}{|c|c|c|c|c|}
\hline $\begin{array}{l}\text { Amiodarone } \mathrm{m} / \mathrm{z} \\
646.00 \text { ion counts } \\
\text { before matrix } \\
\text { deposition (MD) }\end{array}$ & $\begin{array}{l}\text { Amiodarone } \mathrm{m} / \mathrm{z} \\
646.00 \text { ion counts } \\
\text { after matrix } \\
\text { deposition (MD) }\end{array}$ & $\begin{array}{l}\text { Enhancement ratio } \\
\text { peak area (after } \\
\text { MD/before MD) }\end{array}$ & $\begin{array}{l}\text { Scans range before } \\
\text { MD }\end{array}$ & Scans range after MD \\
\hline 23760 & 63640 & 2.7 & $29-34$ & $69-74$ \\
\hline 21960 & 60790 & 2.8 & 94-99 & 134-139 \\
\hline 21190 & 54870 & 2.6 & $159-164$ & 199-204 \\
\hline 20600 & 55910 & 2.7 & $224-229$ & $264-269$ \\
\hline 20460 & 56620 & 2.8 & $289-294$ & $329-334$ \\
\hline 20260 & 54360 & 2.7 & 354-359 & 394-359 \\
\hline 20270 & 54930 & 2.7 & $419-424$ & $459-464$ \\
\hline 19770 & 54300 & 2.7 & $484-489$ & $524-529$ \\
\hline 19500 & 50540 & 2.6 & $549-554$ & $589-594$ \\
\hline 19680 & 53950 & 2.7 & 614-619 & $654-659$ \\
\hline 19990 & 48230 & 2.4 & $679-684$ & $719-724$ \\
\hline 19960 & 50610 & 2.5 & $744-749$ & 784-789 \\
\hline 19530 & 51000 & 2.6 & $809-814$ & $849-854$ \\
\hline 20100 & 52310 & 2.6 & 874-879 & 914-919 \\
\hline \multirow[t]{3}{*}{20470} & 51560 & 2.5 & $939-945$ & $979-985$ \\
\hline & Average & 2.6 & & \\
\hline & RSD (\%) & 3.9 & & \\
\hline
\end{tabular}




\section{Experimental Details}

Sputter Distribution of Matrix. Two experiments were performed for the purpose of determining the distribution of collected deposits. Top-mount sample structures (cf. Figs. 1c and 1d) were utilized in both experiments performed at room temperature. Clean silicon wafers of 10 $\mathrm{mm} \times 10 \mathrm{~mm}$ dimensions were used as collector surface. The SIMS instruments were operated without charge compensation. Ellipsometry mapping was utilized to create thickness maps of the organic deposits on the silicon wafer. In the first experiment, a silicon wafer was PVD coated with Irganox $1010(\sim 1.7 \mu \mathrm{m}$ thick layer, $c f$. Fig. SI-4 for chemical structures), cut to about $10 \mathrm{~mm} \times 2.3$ $\mathrm{mm}$ size and mounted on top of the matrix reservoir surface using double sided tape. The added height of the silicon wafer supported matrix layer as compared to directly on the metal surface applied matrices was accounted for by mounting the collector silicon wafer on top of another silicon wafer of the same height. The geometrical deviation from the ME SIMS experiment was minimal. The $10 \mathrm{keV} \mathrm{Ar} 5000$ beam of a TOF-SIMS IV instrument (1.6 nA current) (200 $\mu$ s cycle time) was set to a raster of $1 \mathrm{~mm} \times 1 \mathrm{~mm}$ (at sample height) and directed onto the Irganox 1010 surface. The instrument was operated in non-interlaced mode for a total erosion time of $140 \mathrm{~min}$. Optical interrogation after the sputtering experiment revealed a crater surface area on the elevated and tilted matrix surface of $0.7 \mathrm{~mm} \times 2.0 \mathrm{~mm}$. In a second experiment, $30 \mu \mathrm{L}$ of a 2,5-DHB matrix solution $(649 \mathrm{mmol} / \mathrm{L}$ in $\mathrm{MeOH})$ total was applied in $\sim 2 \mu \mathrm{L}$ aliquots to the matrix reservoir surface using a micropipette, allowing the solvent to completely dry between application steps. The 10 $\mathrm{keV} \mathrm{Ar}_{2500}$ beam of a TOF.SIMS 5 instrument (7.0 nA current) was set to a raster of $0.8 \mathrm{~mm} \times 0.8$ $\mathrm{mm}$ (at sample height) and directed onto the 2,5-DHB matrix surface. The instrument was operated in non-interlaced mode for a total erosion time of $75 \mathrm{~min}$.

ME SIMS Using 2,5-DHB. A total of $50 \mu \mathrm{L}$ of a 2,5-DHB solution $(649 \mathrm{mmol} / \mathrm{L}$ in $\mathrm{MeOH})$ was applied in $\sim 2 \mu \mathrm{L}$ aliquots to the matrix reservoir surface of a top-mount sample structure (cf. Figs. 1c and d) using a micropipette, allowing the solvent to completely dry between application steps. A sample of chloroquine, haloperidol, and amiodarone in tissue homogenate was prepared as described above and used at room temperature. The silicon wafer was cut to bring the region of the sample material closer to the matrix reservoir. The $10 \mathrm{keV} \mathrm{Ar}_{2500}$ beam of a TOF.SIMS 5 instrument (7.0 nA current) was set to a raster of $0.8 \mathrm{~mm} \times 0.8 \mathrm{~mm}$ (at sample height). The beam was centered with the analysis beam field of view for erosion cycles of $60 \mathrm{~s}$ duration each, for initial sample clean-up and for sample clean-up following ME SIMS analysis. The beam was directed at the 2,5-DHB matrix surface for in operando matrix sputtering for 15 min total sputtering time. The TOF-SIMS instrument was operated in positive ion mode with a $30 \mathrm{keV} \mathrm{Bi}_{3}{ }^{+}$ LMIG bunched analysis beam ( $0.15 \mathrm{pA}$ current), a field of view of $500 \mu \mathrm{m} \times 500 \mu \mathrm{m}$ and a raster of $256 \times 256$ pixels, $200 \mu$ s cycle time, and an electron floodgun for charge compensation was used. Interlaced 3D imaging mode was used for matrix sputter deposition.

In situ ME SIMS Depth Profile. The in situ ME-SIMS depth profile was done on the sample of amiodarone in tissue homogenate (prepared as described above) and by using 2,5-DHB as the matrix (prepared as described above). The TOF-SIMS instrument was operated in positive ion mode with a $\mathrm{Bi}_{3}{ }^{+}$LMIG bunched analysis beam ( $0.15 \mathrm{pA}$ current), a field of view of $250 \mu \mathrm{m} \times$ $250 \mu \mathrm{m}$ and a raster of $128 \times 128$ pixels, $200 \mu$ s cycle time, and an electron floodgun for charge compensation was used. Interlaced 3D imaging mode was used for matrix sputter deposition. The $10 \mathrm{keV} \mathrm{Ar}_{2500}$ beam of a TOF.SIMS 5 instrument ( $8.0 \mathrm{nA}$ current) was set to a raster of $0.5 \mathrm{~mm} \times$ 
$0.5 \mathrm{~mm}$ (at sample height). To achieve in situ ME-SIMS 3D imaging and depth profile, the deflection potential in $Y$ direction (parameter YTarget) of the GCIB beam was toggled between three values for sputter cleaning (YTarget $\approx 0 \%$ ), 2D image only (YTarget $\approx+100 \%$ ) and matrix sputtering (YTarget $=-100 \%$ ). This YTarget value was automatically adjusted during the running data acquisition, and synchronized with the progress of the experiment based on the reading of the current number of scans, using the software tool AutoHotkey (GPL license). One cycle of in situ ME-SIMS includes 15 scans sputter cleaning, 10 scans 2D imaging, 30 scans matrix sputtering and 10 scans 2D imaging after matrix deposition.

ME SIMS Multimodal Utilization of 2,5-DHB and $\boldsymbol{\alpha}$-CHCA. Emulsions of 2,5-DHB (6.5 $\mathrm{mol} / \mathrm{L}$ in $\mathrm{MeOH})$ and $\alpha-\mathrm{CHCA}(5.3 \mathrm{~mol} / \mathrm{L}$ in $\mathrm{MeOH})$ were used to apply the two matrices to the reservoir surface of a top-mount sample structure (cf. Figs. 1c and 1d). Both compounds were applied with minimal contact/overlap in adjacent rectangular areas on the matrix reservoir, separated in $X$ direction (cf. Fig. 1). A clean silicon wafer of $10 \mathrm{~mm} \times 10 \mathrm{~mm}$ dimensions was used as sample surface at room temperature. One central analysis location was defined on the sample in line with the dividing line between both matrices, and two sputter positions at distances of $\Delta X= \pm 0.75 \mathrm{~mm}$. The $10 \mathrm{keV} \mathrm{Ar}_{2500}$ beam of a TOF.SIMS 5 instrument (7.0 nA current) was set to a raster of $0.8 \mathrm{~mm} \times 0.8 \mathrm{~mm}$ (at sample height) and its deflection potential in $Y$ direction (parameter YTarget) toggled between two values for either sputter cleaning (YTarget $\approx 0 \%$ ) or matrix sputtering (YTarget $=-100 \%$ ). The TOF-SIMS instrument was operated in negative ion mode with a $30 \mathrm{keV} \mathrm{Bi}_{3}{ }^{+}$LMIG bunched analysis beam (0.15 pA current), a field of view of 500 $\mu \mathrm{m} \times 500 \mu \mathrm{m}$ and a raster of $256 \times 256$ pixels, $200 \mu$ s cycle time, and charge compensation disabled. Erosion cycles were performed in interlaced 3D imaging mode.

ME SIMS Using Volatile Matrices. Two separate experiments were performed under reduced temperature conditions to enable the vacuum introduction of volatile species. For both experiments, a sample of chloroquine, haloperidol, and amiodarone in tissue homogenate was prepared and cooled in the load-lock following the procedure described above. A sample structure to be mounted on a heating and cooling holder was used ( $c f$. Figs. 1e and 1f). The $10 \mathrm{keV} \mathrm{Ar}_{5000}$ beam of a TOF-SIMS IV instrument ( $1.6 \mathrm{nA}$ current) $(200 \mu$ s cycle time) was centered with the analysis beam field of view for simultaneous in operando matrix sputtering ( $c f$. Results and Discussion section for details). The beam was partially directed at the matrix surface for in operando matrix sputtering in interlaced 3D imaging mode. The TOF-SIMS instrument was operated in positive ion mode with a $25 \mathrm{keV} \mathrm{Bi}_{3}{ }^{+} \mathrm{LMIG}$ bunched analysis beam ( $0.12 \mathrm{pA}$ current), a field of view of $500 \mu \mathrm{m} \times 500 \mu \mathrm{m}$ and a raster of $256 \times 256$ pixels, $200 \mu$ s cycle time, and charge compensation enabled. In the first experiment, a $10 \%$ solution of formic acid in water was used to cover the matrix reservoir surface using a micropipette. The sample was analyzed at $-120 \pm 2$ ${ }^{\circ} \mathrm{C}$ (and moderately reduced temperature due to imperfect thermal insulation). A GCIB raster of $0.6 \mathrm{~mm} \times 0.6 \mathrm{~mm}$ (at sample height) was used for a total sputtering time of $10 \mathrm{~min}$. In a second experiment, a 3-NBN solution ( $115 \mathrm{mmol} / \mathrm{L}$ in $\mathrm{MeOH}$ ) was applied in $\sim 2 \mu \mathrm{L}$ aliquots to the matrix reservoir surface using a micropipette, allowing the solvent to completely dry between application steps. The sample was analyzed at $-108 \pm 2{ }^{\circ} \mathrm{C}$ (and moderately reduced temperature due to imperfect thermal insulation). The silicon wafer was cut to bring the center region of the sample material closer to the matrix reservoir. A GCIB raster of $0.75 \mathrm{~mm} \times 0.75 \mathrm{~mm}$ (at sample height) was used for a total sputtering time of $5 \mathrm{~min}$. 